\title{
A reflective intensity modulated fiber tilt angle sensor based on an all-photonic crystal fiber interferometer
}

\author{
Yong Zhao ${ }^{1,2, *}$, Xue-gang $\mathrm{Li}^{1}, \mathrm{Lu} \mathrm{Cai}^{1}$ \\ ${ }^{1}$ College of Information Science and Engineering, Northeastern University, \\ Shenyang 110819, China \\ ${ }^{2}$ State Key Laboratory of Synthetical Automation for Process Industries, Shenyang \\ 110819, China \\ *Corresponding author: zhaoyong@ise.neu.edu.cn
}

\begin{abstract}
A highly sensitive tilt angle sensor based on an all-photonic crystal fiber interferometer (All-PCFI) was proposed and demonstrated in this paper. The All-PCFI was formed by a PCF with two collapse regions that were fabricated by fusion discharge technique. Then a device formed by cantilever beams and iron ball was designed to transform the tilt angle to the strain, which would be detected by measuring the spectral responses of the All-PCFI. Experimental results showed that the spectrum would be red-shifted when the tilt angle changed from 0deg to 90deg. And in the measurement range of $0 \mathrm{deg} \sim 45 \mathrm{deg}$, the linear measurement sensitivity could be up to $55.67 \mathrm{pm} / \mathrm{deg}$. In addition, the sensor could measure the tilt angle in all directions by demodulating signals of the four cantilever beams.
\end{abstract}

Keywords: photonic crystal fiber interferometer tilt angle sensor

\section{Introduction}

Tilt sensing is an important process in many engineering, military fields and aerospace. Therefore, the tilt sensor has been widely studied. All-fiber sensing merits are well known to be small, to transmit over long distance, to be immune to Electro Magnetic Interference (EMI), which are also especially suitable for tilt sensing applications. The previously proposed optical fiber tilt sensors were almost based on fiber Bragg grating [1-6]. In those references, the tilt angle were determined by demodulating shifts of the fiber Bragg grating (FBG) center wavelength when the tilt angle changed. However, the methods used FBG were not perfect because the temperature cross sensitivity issue and the complex production process of the FBG. Therefore, In 2014, Lee C L, et al. proposed a new in-line Mach-Zehnder (M-Z) 
interferometer which was fabricated by a SMF with two tapers to measure the directional tilt and the sensitivity could reach to $335 \mathrm{pm} / \mathrm{deg}$ [7]. But the temperature cross sensitivity issue still existed. In addition, the proposed sensor was fragile and could only measure the directional tilt angle, which usually could not meet the needs of the project. Therefore, a kind of low-sensitive temperature, strong and all directional tilt angle sensor was badly needed. As everyone known, photonic crystal fiber (PCF) had been widely studied because its unique properties especially low temperature sensitivity, which was caused by a very low thermal expansion coefficient (TEC) and low thermo-optics coefficient [8]. And the investigators have been illustrated a series of in-line PCFIs formed by splicing two single mode fibers (SMFs) in the PCF and applied to the measurement of various parameters [9-12]. Meanwhile, the All-PCFI was proposed and applied to sensing field [13-14].

In this paper, a strong, low-sensitive temperature and highly sensitive tilt angle sensor based on a reflective intensity modulated All-PCFI was proposed and demonstrated. The proposed All-PCFI was formed by fabricating two collapse regions in the PCF through fusion discharge technique. And then the end of the second collapse region was plated with the silver film to increase the reflective intensity. The sensor had a smaller size due to the reflection type structure. Meanwhile, the proposed sensor was strong, low-sensitive to temperature and easy to fabricate.

\section{Operating Principle}

\subsection{Operating principle of the All-PCFI}

Fig. 1(a) showed the schematic diagram of the proposed reflected All-PCFI, which was formed by a PCF with two collapse regions fabricated by fusion discharge technique, as shown in Fig. 1(b) and Fig. 1(c).

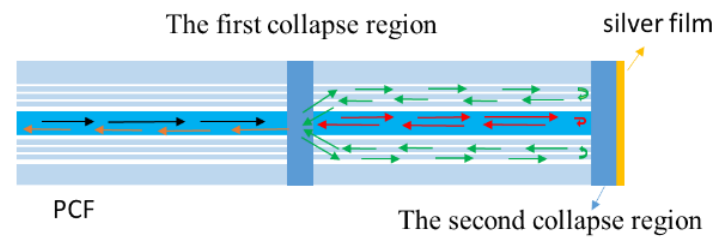

(a)

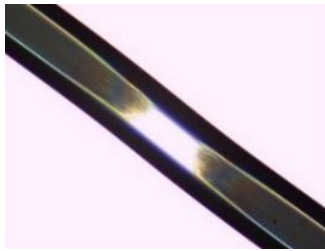

(b)

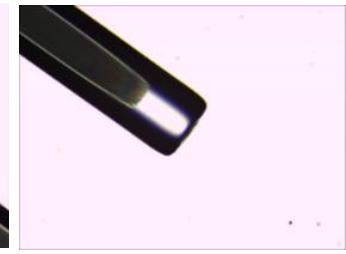

(c)

Fig. 1(a) schematic diagram of All-PCFI (b) the first collapse region of the PCF under the microscope (c) the second collapse region of the PCF under the microscope

The PCF with two collapse regions forms an M-Z interferometer. The input optical signal launches into the PCF and propagates in the core of the PCF with the fundamental mode and the effective refractive index was 1.4475665, as shown in Fig. 
2 (a). When the light propagate through the first collapse region, a part of lights excite the cladding modes, as shown in Fig.2(b) and the others continue to propagate forward along the core of PCF with the fundamental mode. Fig. 2(c) was the optical field distribution when the lights through the first collapse region, which proved that the cladding modes have been excited.

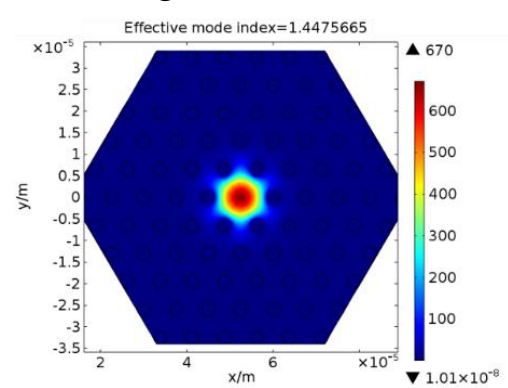

(a)

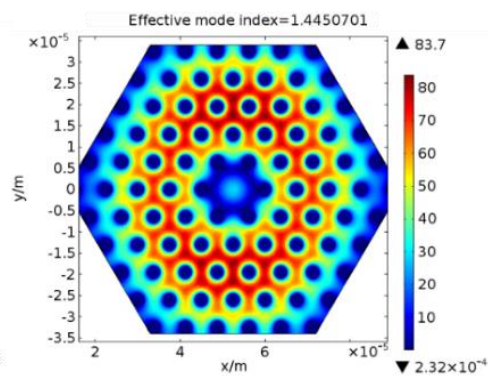

(b)

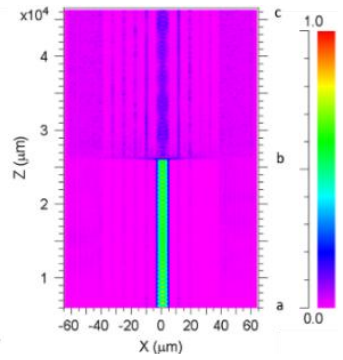

(c)

Fig. 2 (a) the fundamental mode of the PCF (b) one of the cladding modes (c) the electric field distribution when the light through the first collapse region

When the lights (core mode and cladding modes) arrive to the second collapse region and silver film, the lights were reflected and returned back. Then the reflected cladding modes would re-coupling back to the core of the PCF and interfere with the reflected core mode light in the first collapse region. Because of the phase difference between the core mode and the cladding modes, an interference output could be observed, as shown in Fig. 3. Fig.3 was the spectrum simulated by Rsoft, which proved the existence of $\mathrm{M}-\mathrm{Z}$ interference. It is noted that the interference spectrum was uneven, which may be caused by stacking multiple cladding modes.

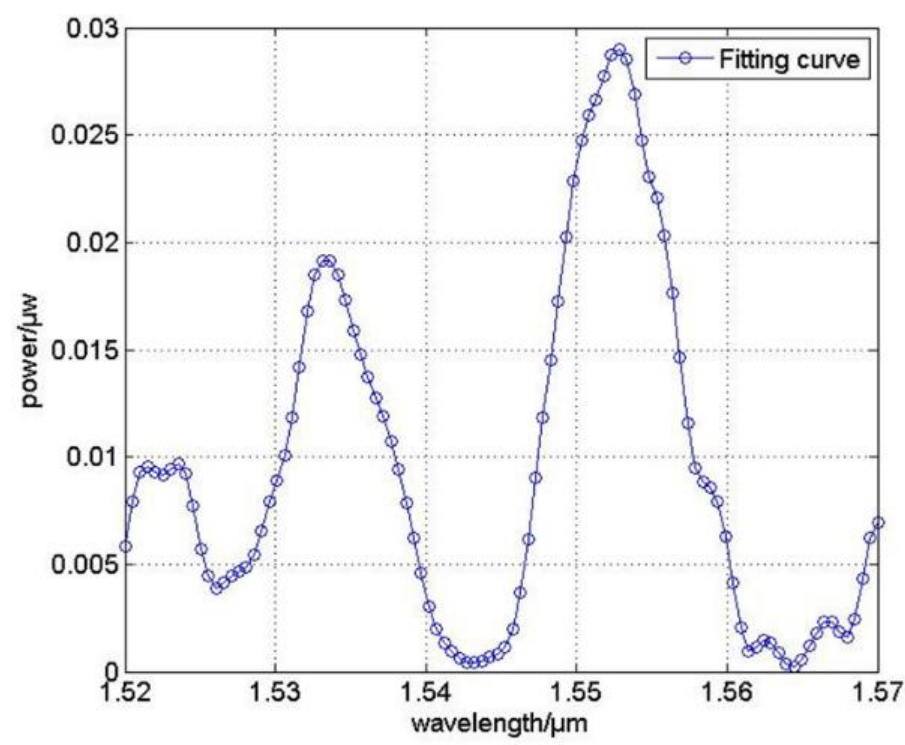

Fig. 3 the simulation spectrum of the All-PCFI

The interference spectrum could be analyzed by using a simple two-mode interference model, which had been widely used in this field to qualitatively analyze 
the fiber mode interference:

$$
I_{\text {out }}=I_{\text {core }}+I_{\text {cladding }}+2 \sqrt{I_{\text {core }} I_{\text {cladding }}} \cos \delta
$$

where $I_{\text {core }}$ and $I_{\text {cladding }}$ were light intensities of the core and cladding modes, and $\delta$ was the phase difference between cladding modes and core mode, which could be expressed as

$$
\delta=\frac{2 \pi L}{\lambda} \times \Delta n_{n e f f}
$$

where $\lambda$ was the centre wavelength of the light and the $\Delta n_{\text {neff }}$ was the effective index difference, which could be defined as:

$$
\Delta n_{\text {neff }}=n_{\text {core }}^{\text {neff }}-n_{\text {clad }}^{\text {neff }}\left(n_{\text {core }}^{\text {neff }}>n_{\text {clad }}^{\text {neff }}\right)
$$

When the PCF was bent or stretched, photo-elastic effect would happen and cause the refractive index profile of the fiber changed. Thus the RI difference, i.e. $\Delta n_{\text {neff }}$ was changed.

It could be seen from the Equ.1 that the interference light intensity got the minimum when the phase difference $\delta$ was an odd multiple of $\pi$ and it could be expressed as

$$
(2 K+1) \pi=\frac{2 \pi L}{\lambda} \times \Delta n_{\text {neff }}
$$

Where the $K$ was the natural number. And the dip wavelength of the interference spectrum could be expressed as

$$
\lambda_{\min }=\frac{2 L \times \Delta n_{n e f f}}{2 K+1}
$$

Therefore, when the $\Delta n_{\text {neff }}$ changed, the dip wavelength of the interference spectrum would change. Above analysis showed that the strain could be measured by recording the wavelength shift of the interference spectrum recorded by optical spectrum analyser (OSA).

\subsection{Operating principle of the tilt angle measuring device}

Fig. 4 was the tilt angle measuring device, which transformed the tilt angle to the strain of the cantilever beam. The device was formed by an iron ball, four cantilever beams and the four supports mainly. The strain of the cantilever beam would change with the tilt angle $\theta$ due to the gravitational effects. The sensing fiber was pasted in 
the cantilever beam to measure the strain.

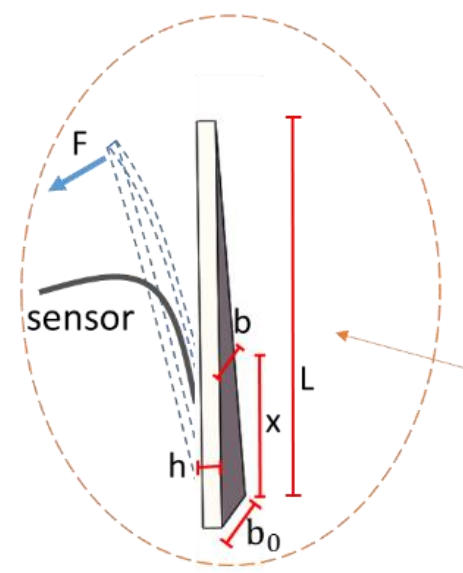

(a)

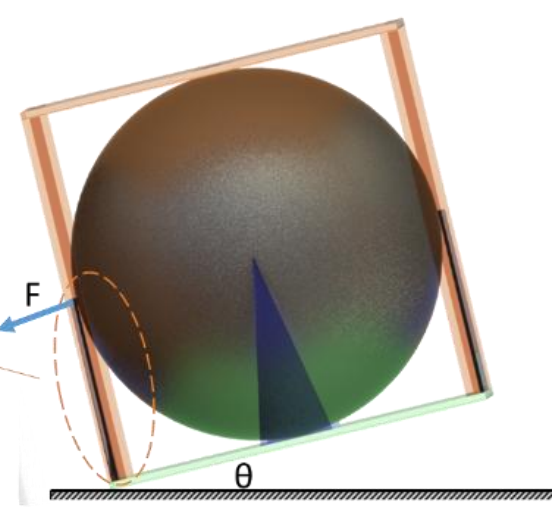

(b)

Fig. 4(a) schematic diagram of the cantilever beam changing when the device has a tilt angle (b) the device transformed the tilt angle to the strain

In order to establish the relationship between the tilt angle $\theta$ and strain, the central axis strain of the cantilever beam needed to be analyzed when the free end of the cantilever beam was applied force. Strain was defined as the ratio of the cantilever beam length variation and the original length. Based on the theory of material mechanics, the longitudinal strain on an equal-thickness cantilever can be expressed as:

$$
\varepsilon_{x}=\frac{6(L-x)}{L^{3}} \cdot h w
$$

where $L$ and $h$ were the original length and thickness of the cantilever beam, $w$ is flexivity. When external force $F$ is applied to the free end of the cantilever beam, $w$ could be defined as:

$$
w=\frac{F \cdot L^{3}}{3 E J_{\mathrm{y}}}
$$

where $E$ was the Elastic Modulus of the cantilever beam and $J_{\mathrm{y}}$ was the inertia moment of the cantilever beam section, which could be expressed as:

$$
J_{\mathrm{y}}=\frac{b h^{3}}{12}=\frac{b_{0}(L-x) h^{3}}{12 L}
$$

Where $b$ was the cross section width of the location that has a distance $x$ away from the cantilever beam fixed end and $b_{0}$ was the width. Through the above analysis, the axial strain $\varepsilon_{x}$ could be expressed as: 


$$
\varepsilon_{x}=\frac{6(L-x)}{L^{3}} \cdot h \cdot \frac{12 F L^{3} \cdot L}{3 E b_{0}(L-x) h^{3}}=\frac{24 F L}{E b_{0} h^{2}}
$$

It can be seen from Equ.9 that the axial strain $\varepsilon_{x}$ was proportional to the force $F$ applied in the free end. In this paper, the force $F$ applied in the free end could be expressed as $F=m g * \sin \theta$, where $m$ was the weight of the iron ball and $\theta$ was tilt angle of the whole device, as shown in Fig. 2. Finally, the axial strain $\varepsilon_{x}$ could be expressed as:

$$
\varepsilon_{x}=\frac{24 m g * \sin \theta L}{E b_{0} h^{2}}
$$

Therefore, the strain of the sensing fiber pasted in central axis of the cantilever beam was uniform and would change with the tilt angle $\theta$ of the device.

In this paper, the materials of device were the organic glass and iron. And the structural parameters of the cantilever beam were shown in Tab.1.

Tab.1 parameters of the cantilever beam

\begin{tabular}{|c|c|c|c|c|c|}
\hline$L / \mathrm{cm}$ & $h / \mathrm{mm}$ & $b_{0} / \mathrm{cm}$ & $E / \mathrm{Gp}$ & $m / \mathrm{g}$ & radius of iron ball $/ \mathrm{cm}$ \\
\hline 2.5 & 2 & 1 & 4.35 & 500 & 2.5 \\
\hline
\end{tabular}

Then the relationship between axial strain of this cantilever beam and the tilt angle $\theta$ was plotted in the Fig. 5. And the finished device was shown in Fig. 6(a).

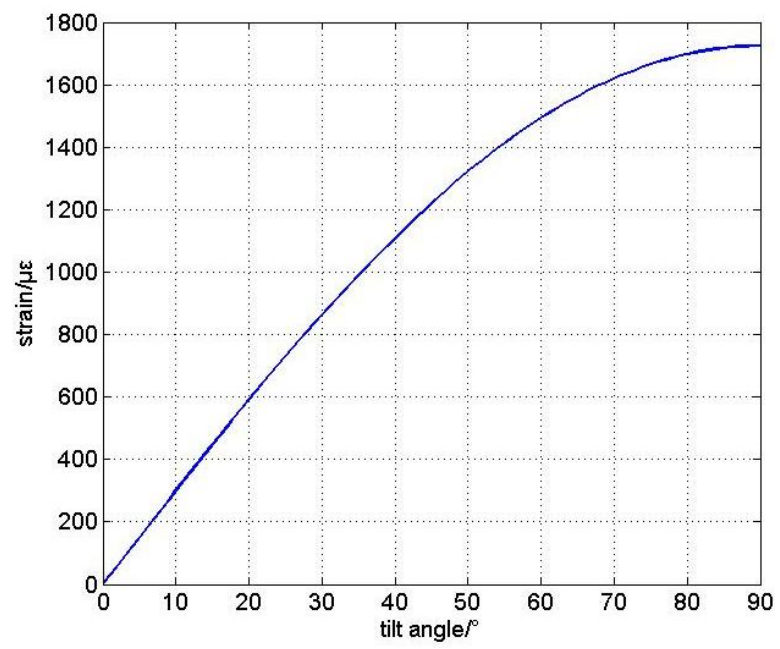

Fig. 5 the relationship curve between the axial strain and tilt angle

According to the upper analysis, the device formed by cantilever beams and iron ball could achieve the purpose of transforming the tilt angle to the strain, which the max strain can reach $1800 \mu \varepsilon$. Meanwhile, the experiment was designed and demonstrated. 


\section{Experiments and Results}

For fabricating the All-PCFI, the PCF (core diameter-9 $9 \mathrm{~m}$, cladding diameter- $125 \mu \mathrm{m}$, air holes diameters-5 $\mathrm{m}$ ) provided by Yangtze Optical Electronic Co., Ltd (China, Wuhan) was employed. The All-PCF interferometer structure was formed by fabricating two collapse regions in the PCF through fusion discharge technique and the micrographs were shown in Fig. 1(b) and Fig. 1(c). Then the end of fiber was plated with a layer of silver film to increase the reflective intensity.

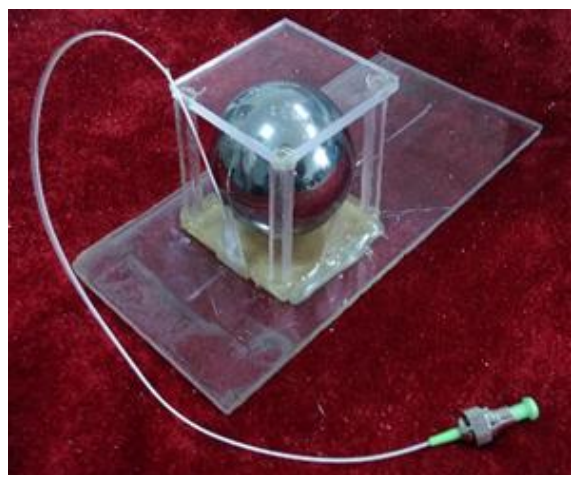

(a)

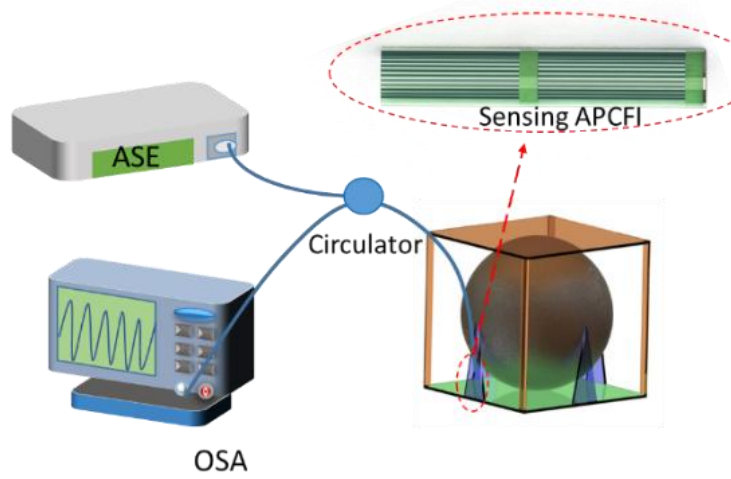

(b)

Fig. 6 (a) the measuring tile angle device and (b) the diagram of experimental system

The experiment system for investigating the tilt angle sensing properties of the sensor was shown in Fig. 6(b). It consisted of a broadband amplified spontaneous emission source (ASE, wavelength ranging from 1520 to $1570 \mathrm{~nm}$ ), a tilt angle measuring device and an optical spectrum analyzer (OSA, AQ6370). When a broadband light source was connected to the input, interference spectra could be observed. Fig. 7 showed the spectra of the different interference arm lengths (i.e. the length between the two collapse regions). It could be seen from the Fig. 7 that the number of interference dips increased with the interference arm length. The length of interference arm length in the experiment was $2 \mathrm{~cm}$, due to the suitable period of the spectrum and the limit of the cantilever beam length.

The sensing fiber was pasted in the cantilever beam to measure tilt angle. The tilt angle could be adjusted from $0^{\circ}$ to $90^{\circ}$. Experimental results showed that the spectrum would red shift with the increasing of the tilt angle, as shown in Fig. 8(a). By measuring the shifts of dips wavelength with an OSA, the tilt angle could be obtained. When the tilt angle was $0^{\circ}$, one of the dips occurred at $1557 \mathrm{~nm}$ and red shift with the increasing of tilt angle. When the tilt angle increased to $90^{\circ}$, a $3.27 \mathrm{~nm}$ wavelength shift of the dip was measured. The characteristic wavelength was extracted and the fitting curve was plotted, as shown in Fig. 8(b). 


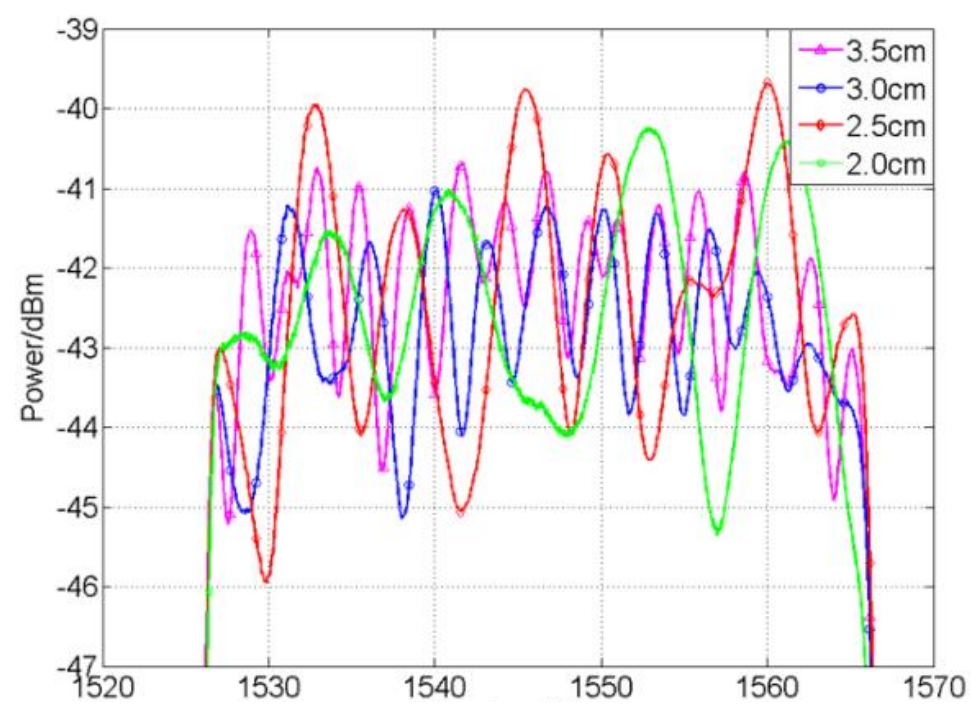

Fig. 7 the spectrums with different interference arm length.

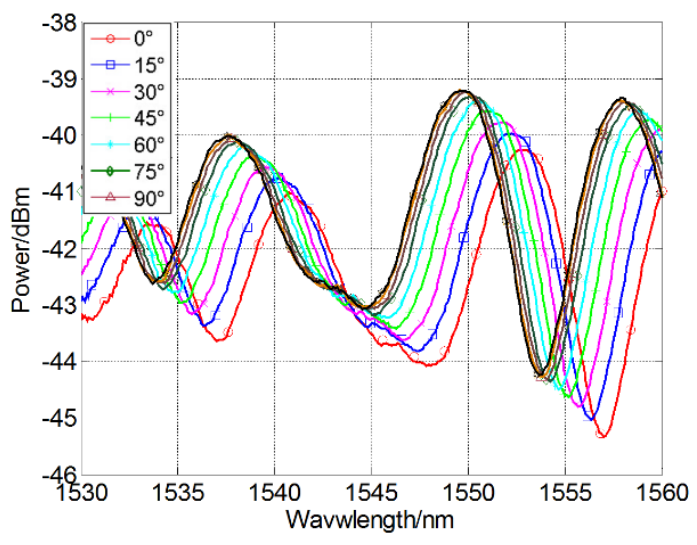

(a)

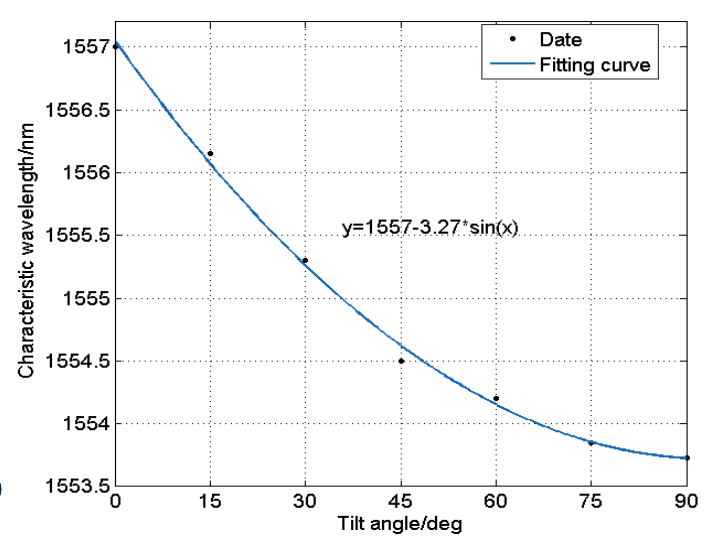

(b)

Fig. 8 experimental results and analysis of the tile angle measurement (a) spectra under different tilt angle (b) fitting curve between wavelengths and tilt angle

Fig. 8(b) showed a sine relationship instead of linear relationship. The reason was that the strain of the cantilever beam was a sine relationship with the tilt angle. In addition, the curve was refitted in the range of $0 \mathrm{deg} \sim 45 \mathrm{deg}$, as shown in Fig. 9, and the repeatability measurement also was implemented.

Fig. 9 showed that the linearity was pretty and the measuring sensitivity of the tilt angle could reach $55.67 \mathrm{pm} / \mathrm{deg}$. Meanwhile, the sensor also had good repeatability. The sensor can measure the tilt angle approaching to 90deg, but the sensitive was quite low. When the tilt angle changed from the $75^{\circ}$ to $90^{\circ}$, only a $0.12 \mathrm{~nm}$ wavelength shift of the transmission minimum was measured. But the linearity and sensitivity were better in the range of $0 \mathrm{deg} 45 \mathrm{deg}$, which could satisfy the requirements of general tilt angle measurement. It is worth mentioning that the proposed sensor could achieve the tilt angle measurement in all directions by demodulating the signals of the four cantilever beams if the sensing fibers were pasted 
in all four cantilever beams.

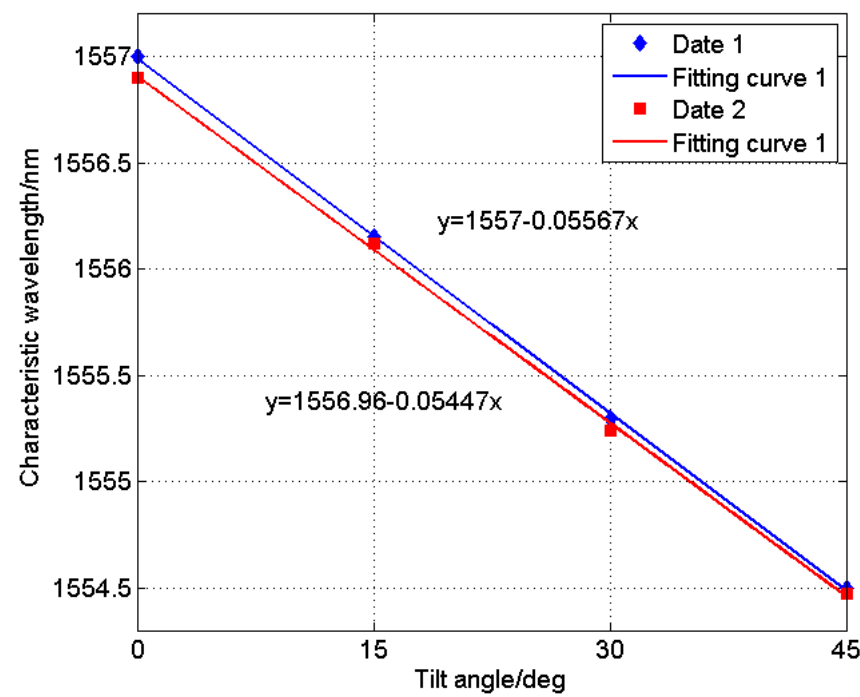

Fig. 9 the dates and fitting curves in the range of $0 \mathrm{deg} \sim 45 \mathrm{deg}$

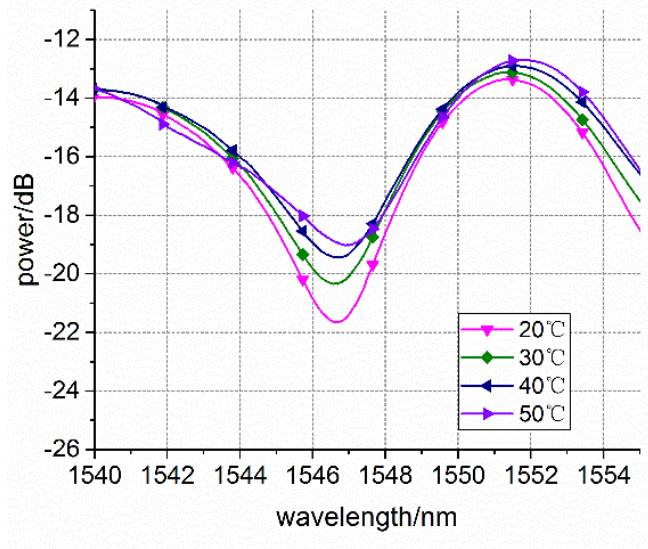

(a)

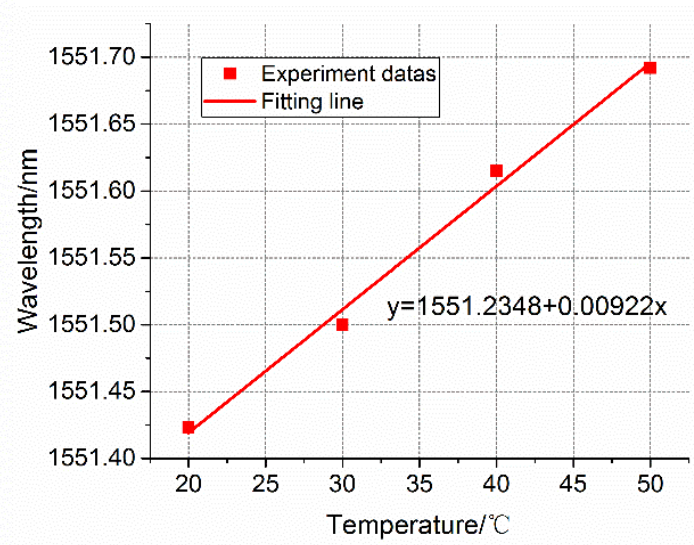

(b)

Fig. 10 (a) spectra with the different temperature and the fitting of the one characteristic wavelength

Considering temperature influences, the silica-based sensors exhibit low temperature sensitivity due to a very low thermal expansion coefficient (TEC) of about $5.5 \times 10^{-7} /{ }^{\circ} \mathrm{C}$ and low thermo-optics coefficient (TOC) of about $5.5 \times 10^{-6} /{ }^{\circ} \mathrm{C}$ [8], which have been further validated by experiment in this paper. Fig. 10(a) was the measured transmission spectra under different temperatures and the fitting line was shown in Fig. 10(b). It can been seen that the proposed sensor was sensitive to temperature, but the temperature sensitivity was pretty low and only was $9.2 \mathrm{pm} /{ }^{\circ} \mathrm{C}$. This meant the equivalent tilt angle change only was $0.165 \mathrm{deg}$ in the range of 0deg 45deg when temperature changed $1{ }^{\circ} \mathrm{C}$.

\section{Conclusion}


A reflective intensity modulated fiber tilt angle sensor based on an All-PCFI formed by a PCF with two collapse regions was proposed and demonstrated. Experimental results showed that the characteristic wavelength of the interference spectrum appeared a sine relationship with the tilt angle in the range of 0deg 90deg. In the measurement range of $0 \mathrm{deg} \sim 5 \mathrm{deg}$, the linear measurement sensitivity can be up to $55.67 \mathrm{pm} / \mathrm{deg}$. Meanwhile, the sensor had the advantages of simple structure, small size, high sensitivity, low cost, low temperature sensitivity and good repeatability. In addition, the proposed sensor could measure tilt angle in all directions by demodulating the signals of the four cantilever beams.

\section{Acknowledgments}

This work was supported in part by the National Science Foundation for Distinguished Young Scholars of China under Grant 61425003, the National Natural Science Foundation of China under Grant 61273059, the Fundamental Research Funds for the Central Universities under Grant N140404021 and N130104002, and State Key Laboratory of Synthetical Automation for Process Industries under Grant $2013 Z C X 09$.

\section{References}

[1] Tian, X. G., \& Tao, X. M. Torsion measurement using fiber Bragg grating sensors[J]. Experimental mechanics, 2001, 41(3): 248-253.

[2] Xiaoyong, F., \& Maosheng, C. Theoretical analysis of 2D laser angle sensor and several design parameters[J]. Optics \& Laser Technology, 2002, 34(3): 225-229.

[3] Guan, B. O., Tam, H. Y., \& Liu, S. Y. Temperature-independent fiber Bragg grating tilt sensor[J]. Photonics Technology Letters, IEEE, 2004, 16(1): 224-226.

[4] Peng, B. J., Zhao, Y., Zhao, Y., \& Yang, J. Tilt sensor with FBG technology and matched FBG demodulating method[J]. Sensors Journal, IEEE, 2006, 6(1): 63-66.

[5] Au, H. Y., Khijwania, S. K., Fu, H. Y., Chung, W. H., \& Tam, H. Y. Temperature-insensitive fiber Bragg grating based tilt sensor with large dynamic range[J]. Journal of Lightwave Technology, 2011, 29(11): 1714-1720.

[6] Munendhar, P., Aneesh, R., \& Khijwania, S. K. Development of an all-optical temperature insensitive nonpendulum-type tilt sensor employing fiber Bragg gratings[J]. Applied optics, 2014, 53(16): 3574-3580.

[7] Lee, C. L., Shih, W. C., Hsu, J. M., \& Horng, J. S. Asymmetrical dual tapered fiber Mach-Zehnder interferometer for fiber-optic directional tilt sensor[J]. Optics express, 2014, 22(20): 24646-24654. 
[8] Hsu, J. M., Horng, J. S., Hsu, C. L., \& Lee, C. L. Fiber-optic Michelson interferometer with high sensitivity based on a liquid-filled photonic crystal fiber[J]. Optics Communications, 2014, 331: 348-352.

[9] Choi H Y, Kim M J, Lee B H. All-fiber Mach-Zehnder type interferometers formed in photonic crystal fiber[J]. Optics Express, 2007, 15(9): 5711-5720.

[10] Aref, S. H., Amezcua-Correa, R., Carvalho, J. P., Frazão, O., Caldas, P., Santos, J. L., ... \& Knight, J. C. Modal interferometer based on hollow-core photonic crystal fiber for strain and temperature measurement[J]. Optics express, 2009, 17(21): 18669-18675.

[11] Bai, X., Fan, D., Wang, S., Pu, S., \& Zeng, X. (2014). Strain Sensor Based on Fiber Ring Cavity Laser With Photonic Crystal Fiber In-Line Mach-Zehnder Interferometer[J]. Photonics Journal, IEEE, 2014, 6(4):1-8.

[12] Li, T., Dong, X., Chan, C. C., Hu, L., \& Qian, W. Simultaneous strain and temperature measurement based on a photonic crystal fiber modal-interference interacting with a long period fiber grating[J]. Optics Communications, 2012, 285(24): 4874-4877.

[13] Villatoro, J., Kreuzer, M. P., Jha, R., Minkovich, V. P., Finazzi, V., Badenes, G., \& Pruneri, V. Photonic crystal fiber interferometer for chemical vapor detection with high sensitivity[J]. Optics Express, 2009, 17(3): 1447-1453.

[14] Park, K. S., Choi, H. Y., Park, S. J., Paek, U. C., \& Lee, B. H. Temperature robust refractive index sensor based on a photonic crystal fiber interferometer[J]. Sensors Journal, IEEE, 2010, 10(6): 1147-1148. 


\section{Biographies}

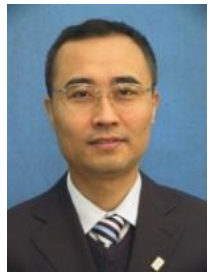

Yong Zhao received the M.A. and Ph.D. degrees, respectively, in precision instrument and automatic measurement with laser and fiber-optic techniques from the Harbin Institute of Technology, Harbin, China, in 1998 and 2001. He was a Postdoctor in the Department of Electronic Engineering of Tsinghua University, from 2001 to 2003, and then was an Associate Professor in the Department of Automation, Tsinghua University of China. In 2006, he was a Visiting Scholar at the University of Illinois in Urbana and Champagne, USA. He is currently a Full Professor with the Northeastern University, Shenyang, China. His current research interests include development of fiber-optic sensors and device, fiber Bragg grating sensors, novel sensor materials and principles, slow light and sensor technology, and optical measurement technologies. He has authored or coauthored more than 180 scientific papers and conference presentations, 10 patents, and 5 books. He is a Member of the Editorial Boards of the international journals such as Sensor Letters, Instrumentation Science and Technology, the Journal of Sensor Technology, and Advances in Optical Technologies.

Prof. Zhao was awarded a first prize scholarship in 2000 by the China Instrument and Control Society and the Sintered Metal Corporation (SMC) scholarship in Japan. He was a scholarship in Japan. In 2008, he was awarded as the "New Century Excellent Talents in University" by the Ministry of Education of China. In 2009, he was awarded as the "Liaoning Bai-Qian-Wan Talents" by Liaoning Province. In 2011, he was awarded by the Royal Academy of Engineering as an Academic Researcher at the City University London. In 2013, he was awarded as the "High Level Talents" by the Northeastern University. He was awarded by the National Science Foundation for Distinguished Young Scholars of China, in 2014.

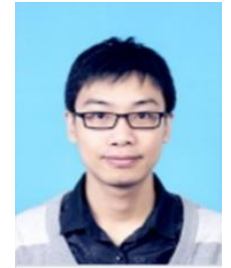

Xuegang Li was born in Hebei, China, in December 1991. He received his B.E. degrees in the College of Information Science and Engineering from the Northeastern University, China, respectively in 2014. He is now a $\mathrm{PhD}$ student of Northeastern University. His research interests are fiber optical sensors, modal interference sensors, in-fiber interferometer and its sensing applications. He has authored and co-authored several scientific papers.

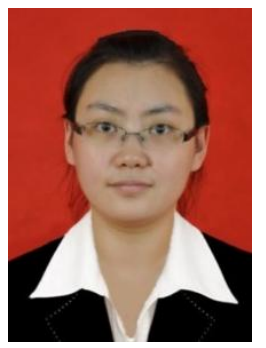

Lu Cai was born in Liaoning, China, in Jul. 1990. She received her B.A. degrees in the College of Information Science and Engineering from the Northeastern University, China, in 2013. She is now a PhD student of Northeastern University. Her research interests are optical fiber sensors, optical modal interference sensors, in-fiber interferometer and its sensing applications. She has authored and co-authored more than ten scientific papers. 